\title{
Common subclinical hypothyroidism during Whipple's disease
}

\author{
Jean-Christophe Lagier ${ }^{1}$, Florence Fenollar ${ }^{1}$, Jacques Chiaroni ${ }^{2}$, Christophe Picard ${ }^{2}$, Christiane Oddoze ${ }^{3}$, \\ Laurent Abi-Rached ${ }^{1,4}$ and Didier Raoult ${ }^{1 *}$
}

\begin{abstract}
Background: Classic Whipple's disease is caused by T. whipplei and likely involves genetic predispositions, such as the HLA alleles $D R B 1^{*} 13$ and $D Q B 1 * 06$, that are more frequently observed in patients. T. whipplei carriage occurs in 2-4\% of the general population in France. Subclinical hypothyroidism, characterized by high levels of TSH and normal free tetra-iodothyronine (fT4) dosage, has been rarely associated with specific HLA factors.

Methods: We retrospectively tested TSHus in 80 patients and 42 carriers. In cases of dysthyroidism, we tested the levels of free-T4 and anti-thyroid antibodies, and the HLA genotypes were also determined for seven to eight patients.

Results: In this study, 72-74\% of patients and carriers were male, and among the 80 patients, 14 (17\%) individuals had a high level of TSH, whereas none of the carriers did $(p<0 \cdot 01)$. In the 14 patients with no clinical manifestations, the T4 levels were normal, and no specific antibodies were present. Four patients treated with antibiotics, without thyroxine supplementation, showed normal levels of TSHus after one or two years. One patient displayed a second episode of subclinical hypothyroidism during a Whipple's disease relapse five years later, but the subclinical hypothyroidism regressed after antibiotic treatment. HLA typing revealed nine alleles that appeared more frequently in patients than in the control cohort, but none of these differences reached significance due to the small size of the patient group.
\end{abstract}

Conclusion: Regardless of the substratum, classic Whipple's disease could lead to subclinical hypothyroidism. We recommend systematically testing the TSH levels in patients with Whipple's disease.

Keywords: Tropheryma whipplei, Whipple's disease, Subclinical hypothyroidism, HLA

\section{Background}

Whipple's disease, first described in 1907, is a chronic infectious disease caused by Tropheryma whipplei $[1,2]$. The positive diagnosis of classic Whipple's disease is based on a positive histological assessment of a small bowel biopsy [1-3]. In addition to classic Whipple's disease, $T$. whipplei also causes localized infections, such as endocarditis or encephalitis [1,4]. Although most individuals can eradicate the bacteria after a primo-infection (gastroenteritis or bacteremia), $[5,6]$ others remain asymptomatic carriers [7], and an even smaller number of individuals develop chronic disease [8]. Genetic predispositions are strongly suspected in classic Whipple's disease because human populations are commonly exposed to T. whipplei

\footnotetext{
* Correspondence: didier.raoult@gmail.com

'Aix Marseille Université, URMITE, UM63, CNRS 7278, IRD 198, INSERM 1095, Faculté de Médecine, 27 Bd Jean Moulin, 13005 Marseille, France Full list of author information is available at the end of the article
}

$[9,10]$, yet individuals rarely develop classic Whipple's disease. Consistently, classic Whipple's disease mainly occurs in European middle-aged populations and has not been described in Sub-Saharan African populations, despite their high exposure to the bacterium [9]. Similarly, patients frequently relapse, and different $T$. whipplei strains can re-infect patients suffering from classic Whipple's disease, suggesting a lifetime susceptibility to this bacterium [11-13]. Interestingly, a recent study highlighted that the $H L A$ alleles $D R B 1 * 13$ and $D Q B 1 * 06$ occurred significantly more frequently in patients with Whipple's disease than in healthy individuals exposed to the bacteria [10].

Among the hypothyroidism substratum, a broad range of genetic defects has been reported, with different levels of clinical consequences ranging from severe congenital hypothyroidism [14-16] to unapparent manifestations in some cases of thyroid-stimulating hormone (TSH)-resistance [16]. Subclinical hypothyroidism is characterized by 
Table 1 Baseline characteristics of patients and carriers (CWD = Classic Whipple's disease)

\begin{tabular}{lll}
\hline & CWD & $\begin{array}{l}\text { Asymptomatic } \\
\text { carriage of } \\
\text { T. whipplei }\end{array}$ \\
\hline Number of cases & 80 & 42 \\
Male & $58(72.5 \%)$ & $31(73.8 \%)$ \\
Mean age, years (range) & $57.15(28-84)$ & $43.58(18-80)$ \\
Frozen serum (stored $<\mathbf{5}$ years) & $39(48.1 \%)$ & $19(46.3 \%)$ \\
$\begin{array}{l}\text { Frozen serum (stored }<\mathbf{5} \text { years } \\
\text { among the higher TSH levels) }\end{array}$ & $6 / 14(43 \%)$ & $\mathrm{NA}$ \\
\hline
\end{tabular}

high TSH concentrations and normal serum thyroid hormones or serum free thyroid hormones. In the NHAES III study performed among US populations, the prevalence of subclinical hypothyroidism was $4 \cdot 3 \%$, associated with factors such as gender, age, body-mass index, and dietary iodine intake [17]. In addition, the prevalence of hyperthyroidism was higher in Europeans than in African Americans, suggesting that genetic factors also affect TSH secretion [17]. Among the causes of subclinical hypothyroidism, chronic lymphocytic thyroiditis (Hashimoto's) represents 60 to $80 \%$ of the cases [17], but genetic factors, such as the $H L A$ allele $H L A-A * 02$, have also been associated with autoimmune thyroid disorders induced through interferon-alpha-therapy in patients with chronic hepatitis $C$, and an association of the HLA A2-B46-DR9 haplotype with autoimmune thyroid dysfunction has also been described $[10,18]$.

Some studies have reported the occurrence of hypothyroidism during Whipple's disease $[2,19,20]$. Interestingly, a case of primary hypothyroidism with clinical manifestations was recently described, showing that the requirement for thyroxine supplementation dramatically and rapidly decreased after the initiation of antibiotic treatment; indeed, supplementation could be stopped after approximately 30 weeks, suggesting that $T$. whipplei directly infects the thyroid [19]. In addition to the apparent capacity of T. whipplei to infiltrate thyroid tissue [19], we hypothesized that the risk of developing subclinical hypothyroidism is also associated with host genetic factors.

Herein, we conducted a retrospective analysis of the TSH concentrations in 122 individuals with either classic Whipple's disease $(n=80)$ or asymptomatic carriage of T. whipplei $(\mathrm{n}=42)$. We also investigated the HLA types in patients suffering from hypothyroidism.

\section{Methods}

\section{Patients}

Since the first culture of $T$. whipplei in 2000, more than 27,000 T. whipplei PCR amplifications [21] have been performed at our research center for the diagnosis of more than 150 patients with classic Whipple's disease [1]. Among these patients, serum was obtained from 80 patients with a clear diagnosis of classic Whipple's disease. As a control, we used 42 patients with asymptomatic carriage of $T$. whipplei for which serum were available [7].

Definition of classic Whipple's disease and asymptomatic carriage of T. whipplei.

A positive diagnosis of classic Whipple's disease was obtained through the histological assessment (PAS staining and/or immunohistochemistry using antibodies specific for $T$. whipplei) of a small bowel biopsy specimen [2,3]. Asymptomatic carriers were defined as patients without clinical manifestations, showing positive T. whipplei PCR amplification from a stool sample [22].

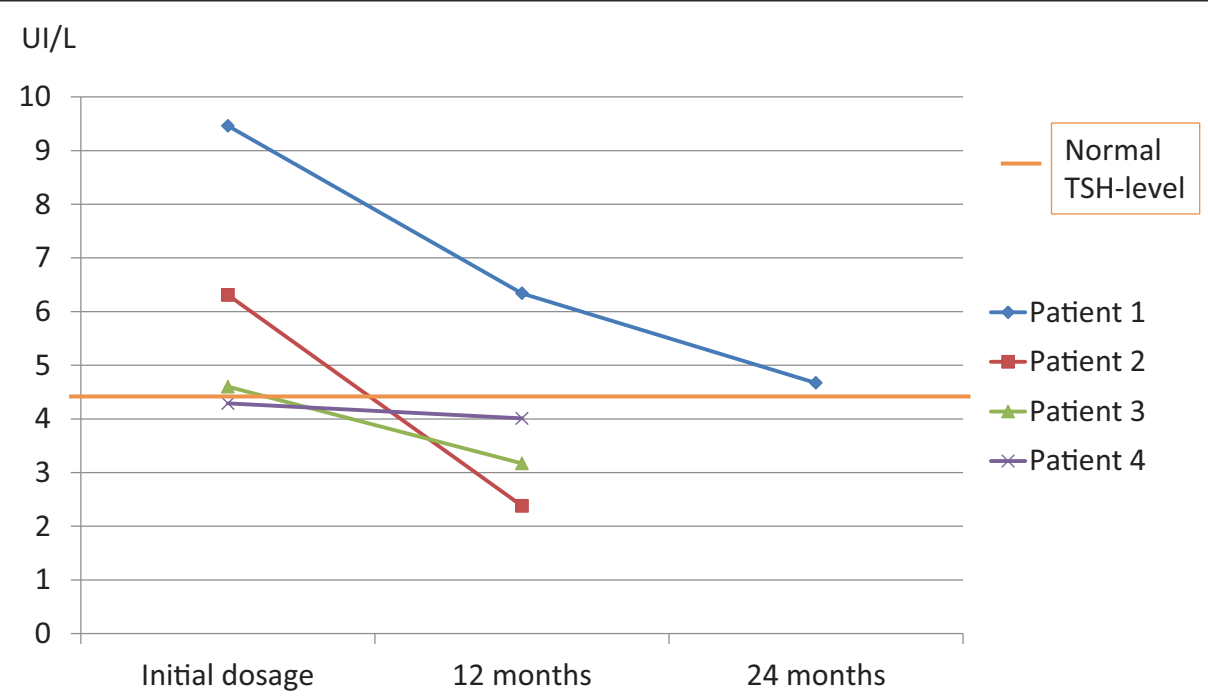

Figure 1 Time evolution of the TSH levels in four patients with CWD treated with antibiotics, without thyroxin supplementation. 


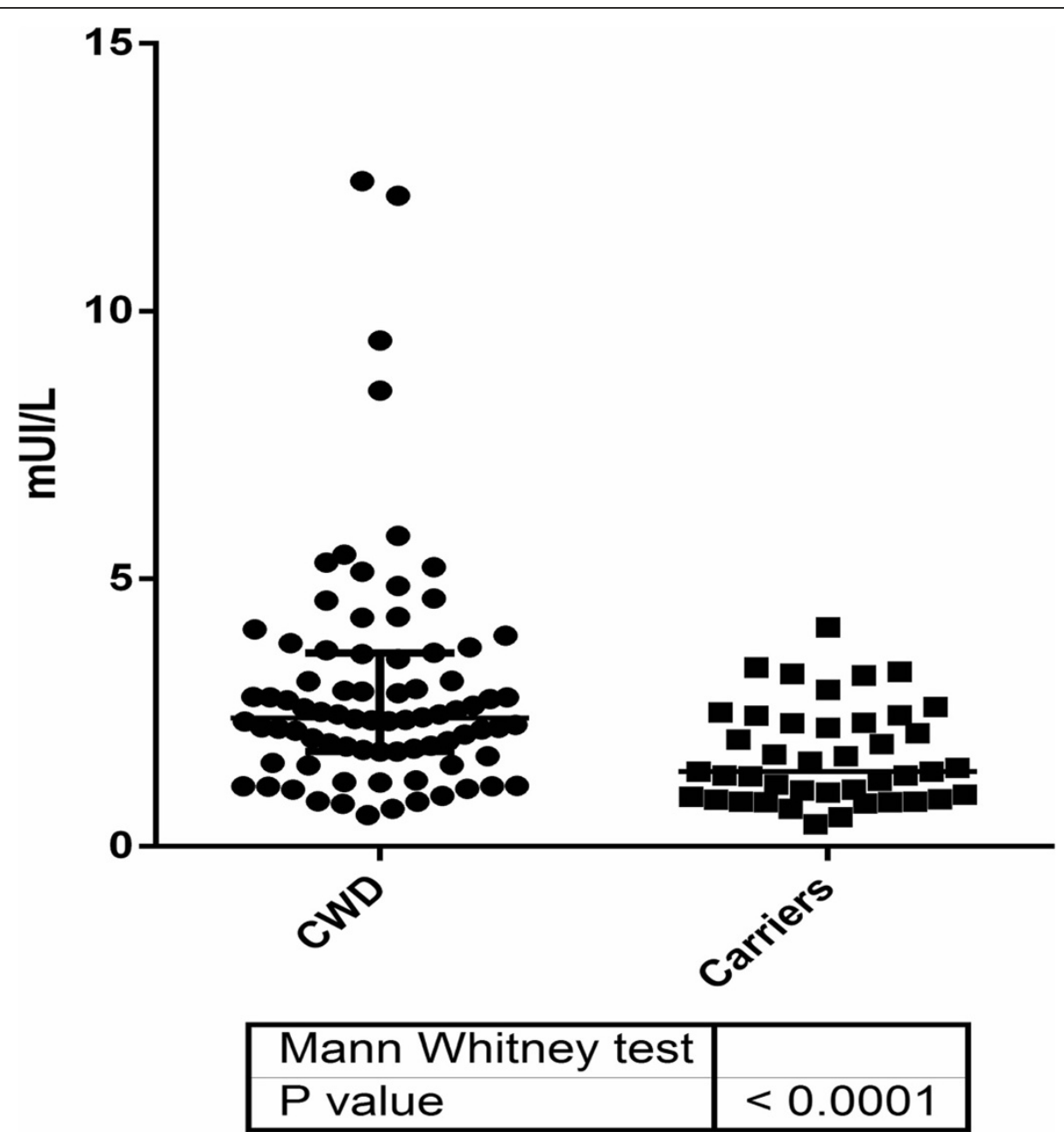

Figure 2 TSH levels in the CWD and asymptomatic carrier groups. The difference between the two groups is significant $(P<0.001)$.

\section{Laboratory findings}

Serum selection and preservation

We tested retrospectively the TSHus levels in serum samples aliquoted and stored at $-80^{\circ} \mathrm{C}$ because of the rarity of $T$. whipplei infections. To ensure that the storage time did not affect our analysis, we selected frozen samples from a wide range of times for the two groups studied: from 2003 to 2011 for asymptomatic carriers and from 2001 to 2012 for classic Whipple's disease patients. The number of samples stored before or within the last five years was approximately equal in the two groups (Table 1).

\section{Serum tests}

The electrochemiluminescence immunoassay (ECLIA) for determination of TSH (thyroid-stimulating hormone), FT4 (free thyroxine hormone), anti-TPO (anti thyroid- specific peroxidase auto antibodies) and anti-Tg (antithyroid-specific thyroglobulin auto antibodies) levels was performed on a Cobas ${ }^{\circledR} 6000<\mathrm{e} 601>$ analyzer (Roche Diagnostics, D-68298 Mannheim, France).

A sandwich immunoassay was used to detect the TSH levels, and a competition immunoassay was used to assess the levels of FT4, anti-TPO and anti-Tg. In these assays, the antigen-antibody complex binds to the solid phase via biotin and streptavidin interactions. The reaction mixture is subsequently aspirated into the measuring cell, where the microparticles are magnetically captured onto the surface of the electrode. The application of a voltage to the electrode induces chemiluminescent emission, measured using a photomultiplier.

Table 2 Factors associated with CWD based on multiple logistic regression analyses

\begin{tabular}{lll}
\hline & OR $(\mathbf{9 5} \% \mathrm{Cl})$ & p-value \\
\hline TSH & $1.846(1.186-2.875)$ & 0.007 \\
Age & $1.058(1.025-1.093)$ & 0.001 \\
\hline
\end{tabular}


Table 3 HLA-A, -B, -C, -DRB1 and -DQB1 types in patients with hypothyroidism

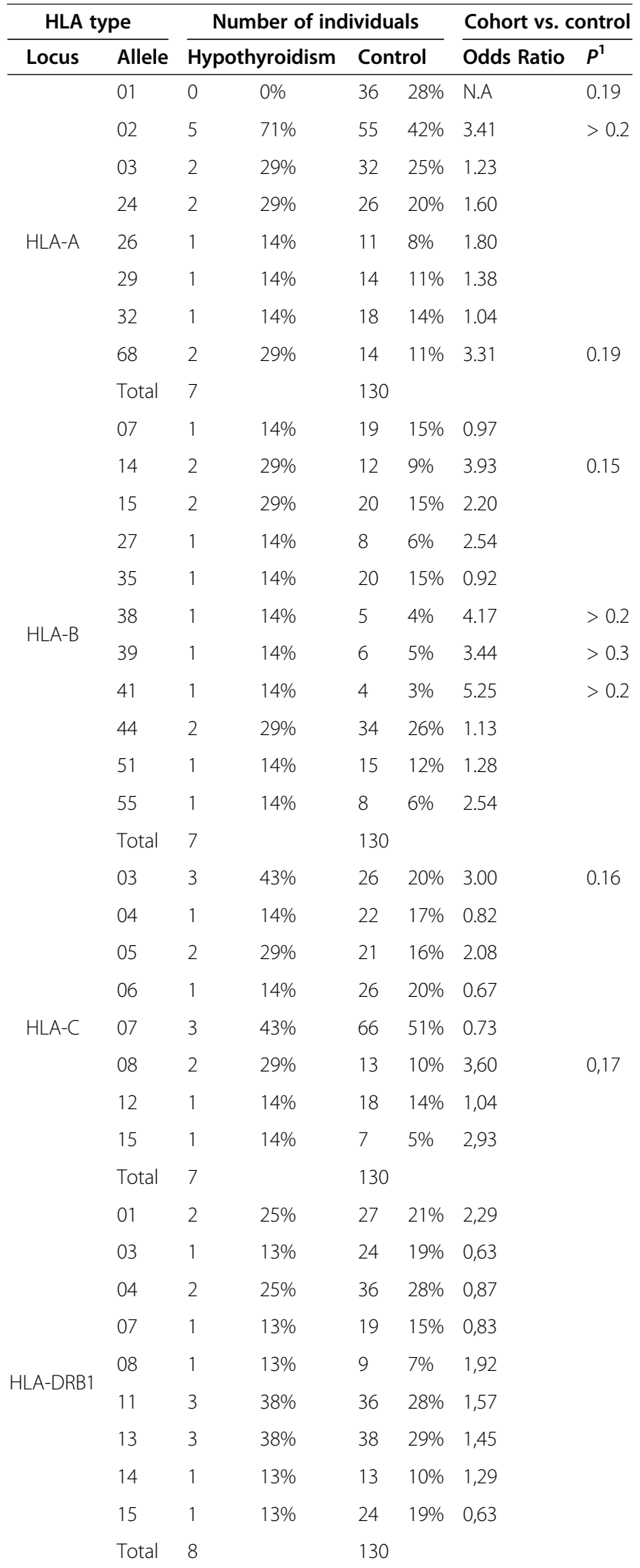

Table 3 HLA-A, -B, -C, -DRB1 and -DQB1 types in patients with hypothyroidism (Continued)

\begin{tabular}{cccccccc}
\hline & 02 & 2 & $25 \%$ & 37 & $29 \%$ & 0,84 & \\
HLA-DQB1 & 03 & 4 & $50 \%$ & 93 & $72 \%$ & 0,40 & $>0.2$ \\
& 04 & 1 & $13 \%$ & 5 & $4 \%$ & 3,57 & $>0.3$ \\
& 05 & 4 & $50 \%$ & 48 & $37 \%$ & 1,71 & \\
& 06 & 3 & $38 \%$ & 56 & $43 \%$ & 0,79 & \\
& Total & 8 & & 130 & & &
\end{tabular}

Odds ratios $\geq 3$ are highlighted in light gray, and odds ratios $<0.5$ are highlighted in dark gray.

1: Two-tailed Fisher Exact Test.

\section{Normal serum level concentration}

The serum level of TSH was normal when the concentration ranged from 0.27 to $4.20 \mathrm{mUI} / \mathrm{L}$. The serum level of T4 was normal when the concentration ranged from 12 to $22 \mathrm{pmol} / \mathrm{L}$. The serum level of anti-thyroid peroxidase antibodies and anti-thyroglobulin antibodies was considered normal for concentrations $<115 \mathrm{UI} / \mathrm{mL}$ and $<34 \mathrm{UI} / \mathrm{mL}$, respectively.

\section{HLA analysis}

Patients and blood donors from southeast France were genotyped for low resolution HLA-A, -B, -C, -DRB1 and $-\mathrm{DQB1}$ loci using LABType SSO Typing Tests (ONE LAMBDA, INC, USA) according to the manufacturer's instructions, and the results were analyzed using HLA Fusion v 1.2.1. software (ONE LAMBDA, INC, USA) for allele identification. The control cohort $(n=130)$ was obtained from southeast France (EFS Rhône Alpes, Histocompatibility Laboratory), and the data were obtained from the Allele frequency net database [23].

\section{Statistical analysis}

Graph pad prism version 6.0 (La Jolla, USA) was used for the statistical analyses shown in Figure 1. Statistical significance was defined as $P<0 \cdot 05$. To exclude possible confounding effects, such as age or gender, a logistic regression model was used. The data analyses were conducted using SPSS v.20 (SPSS Inc., Chicago, IL, USA).

\section{Ethical statement}

Informed consent was obtained from all the participating patients in the study. The study was conducted as part of normal care, without supplementary patient's samples. The study was approved by the Ethics Committee of the Institut Fédératif de Recherche IFR48, Faculty of Medicine, Marseille France (agreement number 13-028).

\section{Results}

Patient's characteristics

Among the 80 patients with classic Whipple's disease and the 42 asymptomatic carriers, $58(72 \cdot 5 \%)$ and 31 
(73.8\%) individuals, respectively, were male, and the mean ages of the patient and carrier groups were $57 \cdot 15$ and $43 \cdot 58$ years, respectively.

\section{Laboratory results}

Among the 80 patients with classic Whipple's disease, 14 individuals (17.5\%) showed high levels of TSH compared with the normal range (range: $4 \cdot 28$ to $12 \cdot 43 \mathrm{mUI} / \mathrm{L}$, mean: $6.58 \mathrm{mUI} / \mathrm{L}$ ) but normal free $\mathrm{T} 4$ concentrations. Among these, 10 were male (71\%) and the mean age was 58.28 years. None of these 14 individuals had another cause of increased TSH or other comorbidity than Whipple's disease. Conversely, none of the 42 asymptomatic carriers showed an increase in the TSH concentration, resulting in a significant difference between the two groups (Figure 2). Using a multiple logistic regression analysis, we showed that TSH is significantly higher in classic Whipple's disease, independent of the age of the patients $(P<0 \cdot 01)$ (Table 2$)$.

Of the 14 patients with both classic Whipple's disease and discrepancies in the TSH serum levels, 13 patients lacked positive anti-thyroglobulin and anti-thyroperoxidase antibodies (the 14th patient could not be tested because of an insufficient amount of serum) excluding a thyroiditis.

\section{Evolution under antibiotic treatment}

We retrospectively tested four patients for the evolution of the TSH level after the initiation of antibiotic treatment for classic Whipple's disease. Three of these four patients were treated with both doxycycline $(200 \mathrm{mg} /$ day $)$ and hydroxychloroquine $(600 \mathrm{mg} /$ day $)$, and the fourth patient was treated with trimethoprim-sulfamethoxazole (320 mg/1600 mg/day); none of these patients was treated with thyroxine supplementation. We observed a dramatic decrease in the TSH levels, particularly in the patient showing the highest TSH levels at the time of diagnosis (Figure 1).
Interestingly, one of the patients experienced a relapse of Whipple's disease at five years after cessation of the antibiotic treatment (Figure 1, Patient 1), and at the time of relapse, this patient also developed subclinical hypothyroidism $(\mathrm{TSH}=7 \cdot 15 \mathrm{UI} / \mathrm{L}$, normal $\mathrm{T} 3$ and $\mathrm{T} 4$ serum levels). Three months after resuming the antibiotic treatment (doxycycline and hydroxychloroquine), the TSH levels were normal, although the patient had not been treated with thyroxin supplementation.

\section{HLA analysis}

Among the 14 patients with classic Whipple's disease and subclinical hypothyroidism, seven individuals were typed for HLA class I, and eight patients were typed for $H L A$ class II (Table 3). A comparison of the HLA frequencies in these patients with those of a control cohort from southeast France [23] revealed that nine alleles were overrepresented in patients (odds ratio $>3$ ), including $H L A-A * 02$, which was observed in 5 of the 7 patients (71\%), but only in 55 of the 130 (42\%) donors in the control group. Despite these large odds ratios, none of these comparisons reached significance due to the small size of the patient group.

\section{Discussion}

Here, we described a significant association between patients suffering from classic Whipple's disease and subclinical hypothyroidism. These results are particularly reliable because we used stringent diagnosis criteria to define classic Whipple's disease, i.e., small bowel histological assessment (Figure 3) [3]. These findings were based on techniques routinely used in biochemical analyses according to the manufacturer's instructions. The influence of the serum preservation in our results was also minimized because we used frozen aliquots of serum stored at $-80^{\circ} \mathrm{C}$, representing the best preservation conditions, and the number of serum samples tested previously or within the last five years was comparable
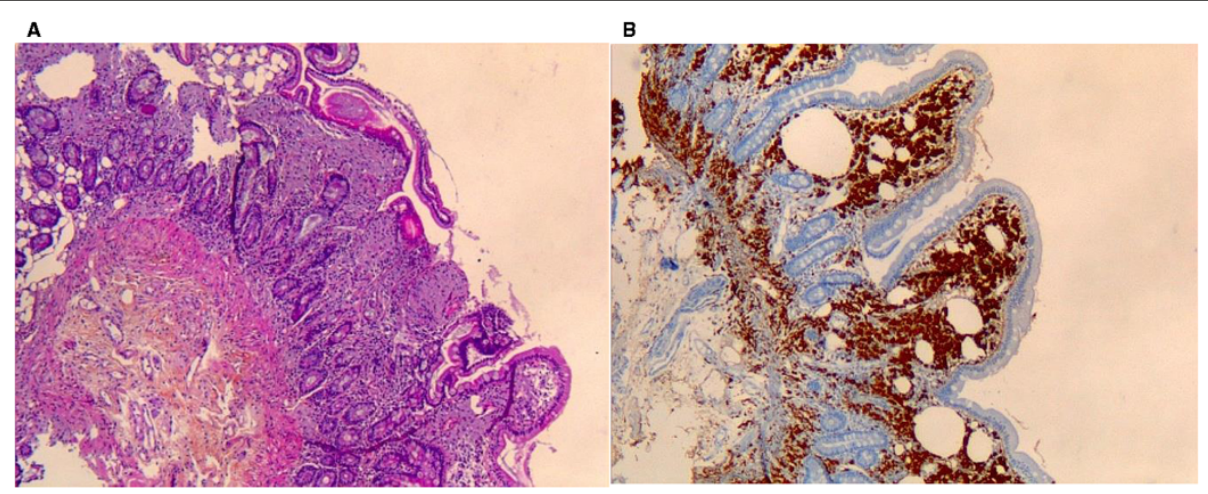

Figure 3 Histological assessment of a duodenal biopsy with positive periodic acid-Schiff staining $(\mathrm{A})$ and positive immunohistochemical staining with polyclonal rabbit anti-T. whipplei antibody and Mayer's haemalum counterstain (B). 
between the patient and carrier groups. Moreover, the proportion of recently sampled or previously stored serum was comparable among the 14 discrepant serum samples (Table 1). The two groups were also comparable in terms of gender proportion with a majority of male as previously known in Whipple's disease [1], but older individuals were present in the patient group compared with the carrier group (Tables 1 and 2). Nevertheless, these results are significant because the multiple logistic regression analysis revealed that the association between higher TSH and classic Whipple's disease is independent of the age of patients (Table 2). Finally, we observed a proportion of patients with subliclinical hypothyroidism (17\%) significantly highest compared with general population (from $3 \%$ to $4 \%$ ) [17].

The characteristics of the symptoms associated with subclinical hypothyroidism, which are neither sensitive nor specific [17] could explain the underdiagnosis of classic Whipple's disease. Despite controversies regarding whether to treat with thyroxin supplementation in these cases, $[17,24,25]$ the early detection of TSH disorders could reveal conditions associated with the risk of developing thyroid disease [17] and consequently an increasing risk of both cardiovascular diseases and heart failure. $T$. whipplei can spread to various tissues and fluids [1,2], leading to thyroid tissue infiltration, and this model is supported by the regression of biological discrepancies under adapted antibiotic treatment [19] and the elimination of other classic causes of subclinical hypothyroidism [17]. However, in previously described cases, thyroid cytology aspirations were PAS-negative, and the $T$. whipplei PCR amplification failed in two different samples [19]. A strong relationship between T. whipplei and subclinical hypothyroidism was demonstrated in a patient showing successive subclinical hypothyroidism during two different disease relapses, which was regressive in both cases after antibiotic initiation.

Hypothyroidism and classic Whipple's disease have been recently associated with specific HLA types. Here, we observed an overrepresentation of nine $H L A$ alleles (HLA-A*02, A*68, B*14, B*38, B*39, B*41, C*03, C*08, DQB1*04) (Table 3) in patients with classic Whipple's disease and subclinical hypothyroidism, but these differences did not reach significance, likely reflecting the small number of patients investigated; thus, a larger cohort could confirm the results of Martinetti et al. [10] regarding HLA and Whipple's disease and will be necessary to confirm these initial observations regarding an association with subclinical hypothyroidism .

\section{Conclusions}

Despite the cause of subclinical hypothyroidism, in the present study, we demonstrated an increased risk in patients with classic Whipple's disease that is not present in T. whipplei asymptomatic carriers. Consequently, we recommend both prospective and systematic testing of the $\mathrm{TSH}$ and T4 serum concentrations in patients with classic Whipple's disease. Because of the subclinical nature and the reversibility under antibiotic treatment, we not recommend a thyroxin supplementation and to control after 1 month of antibiotics.

\section{Abbreviations}

PAS: Periodic acid Schiff; FT4: Free thyroxine hormone; anti-TPO: Anti thyroid-specific peroxidase auto antibodies; anti-Tg: Antithyroid-specific thyroglobulin auto antibodies.

\section{Competing interests}

$J C L, F F, J C, C P, C O, L A R$ and DR declared no conflict of interests. The study was funded by IHU Mediteranee Infection that did not have roles in study design, data collection, analysis, interpretation and writing of the article.

\section{Authors' contributions}

DR designed the study, JCL, LAR and DR wrote the paper; JCL, FF, JC, CP and CO collected the data. JCL, FF, JC, CP, CO, LAR and DR interpreted the data, critically revised and approved the paper.

\section{Authors' informations}

$M D, P h D ~ J C L$ is an infectious disease specialist in $H U$ Méditerranée Infection Marseille France, MD, PhD FF is a clinical microbiologist in IHU Méditerranée Infection, Marseille France, MD, PhD Jacques Chiaroni is the director of Etablissement Français du sang, MD, PhD CP is a biologist, director of HLA-lab in Etablissement Français du sang, PharmD CO is a specialist of biochemistry in Assistance Publique Hopitaux de Marseille, PhD LAR is an immunologist, specialized in the understanding the principles underlying the evolution of vertebrate immune systems, Aix Marseille Université, MD PhD DR is a clinical microbiologist, director of IHU Méditerranée Infection Marseille France.

\section{Acknowledgements}

The authors would like to thank Dr Matthieu Million for assistance with the statistical analysis and Dr Hubert Lepidi for the histological analysis of the small bowel biopsy (Figure 3).

\section{Author details}

${ }^{1}$ Aix Marseille Université, URMITE, UM63, CNRS 7278, IRD 198, INSERM 1095, Faculté de Médecine, 27 Bd Jean Moulin, 13005 Marseille, France. ${ }^{2}$ UMR 7268 (ADES), Aix-Marseille Université, CNRS, EFS, 51 Bd Pierre Dramard, 13916 Marseille, France. ${ }^{3} \mathrm{APHM}, \mathrm{CHU}$ Timone, Laboratoire de Biochimie, 13005 Marseille, France. ${ }^{4}$ Centre National de la Recherche Scientifique, Laboratoire d'Analyse, Topologie, Probabilités - Unité Mixte de Recherche 7353, Equipe ATIP, Aix-Marseille Université, 13331 Marseille, France.

Received: 17 February 2014 Accepted: 30 June 2014 Published: 4 July 2014

\section{References}

1. Lagier JC, Lepidi H, Raoult D, Fenollar F: Systemic Tropheryma whipplei: clinical presentation of 142 patients with infections diagnosed or confirmed in a reference center. Medicine (Baltimore) 2010, 89:337-345. doi:10.1097/MD.0b013e3181f204a8.

2. Fenollar F, Puechal X, Raoult D: Whipple's disease. N Engl J Med 2007, 356:55-66. doi:10.1056/NEJMra062477.

3. Lepidi H, Fenollar F, Gerolami R, Mege JL, Bonzi MF, Chappuis M, Sahel J, Raoult D: Whipple's disease: immunospecific and quantitative immunohistochemical study of intestinal biopsy specimens. Hum Pathol 2003, 34:589-596.

4. Fenollar F, Nicoli F, Paquet C, Lepidi H, Cozzone P, Antoine JC, Pouget J, Raoult D: Progressive dementia associated with ataxia or obesity in patients with Tropheryma whipplei encephalitis. BMC Infect Dis 2011, 11:171. doi:10.1186/1471-2334-11-171.

5. Fenollar F, Mediannikov O, Socolovschi C, Bassene H, Diatta G, Richet H, Tall A, Sokhna C, Trape JF, Raoult D: Tropheryma whipplei bacteremia during fever in rural West Africa. Clin Infect Dis 2010, 51:515-521. doi:10.1086/655677. 
6. Raoult D, Fenollar F, Rolain JM, Minodier P, Bosdure E, Li W, Garnier JM, Richet H: Tropheryma whipplei in children with gastroenteritis. Emerg Infect Dis 2010, 16:776-782. doi:10.3201/eid1605.091801.

7. Fenollar F, Trani M, Davoust B, Salle B, Birg ML, Rolain JM, Raoult D: Prevalence of asymptomatic Tropheryma whipplei carriage among humans and nonhuman primates. J Infect Dis 2010, 197:880-887. doi:10.1086/528693.

8. Greub G: A new piece added to the whipple puzzle: Tropheryma Whipplei primary infection with bacteremia and cough. Clin Infect Dis 2010, 51:522-524. doi:10.1086/655678.

9. Keita AK, Bassene H, Tall A, Sokhna C, Ratmanov P, Trape JF, Raoult D, Fenollar F: Tropheryma whipplei: a common bacterium in rural Senegal. PLoS Negl Trop Dis 2011, 5:e1403. doi:10.1371/journal.pntd.0001403.

10. Martinetti M, Biagi F, Badulli C, Feurle GE, Muller C, Moos V, Schneider T, Marth T, Marchese A, Trotta L, Sachetto S, Pasi A, De SA, Salvaneschi L, Corazza GR: The HLA alleles DRB1*13 and DQB1*06 are associated to Whipple's disease. Gastroenterology 2009, 136:2289-2294. doi:10.1053/j. gastro.2009.01.051.

11. Lagier JC, Fenollar F, Lepidi H, Raoult D: Evidence of lifetime susceptibility to Tropheryma whipplei in patients with Whipple's disease. J Antimicrob Chemother 2011, 66:1188-1189. doi:10.1093/jac/dkr032.

12. Lagier JC, Fenollar F, Lepidi H, Raoult D: Failure and relapse after treatment with trimethoprim/sulfamethoxazole in classic Whipple's disease. J Antimicrob Chemother 2010, 65:2005-2012. doi:10.1093/jac/dkq263.

13. Lagier JC, Fenollar F, Lepidi H, Giorgi R, Million M, Raoult D: Treatment of classic Whipple's disease: from in vitro results to clinical outcome. J Antimicrob Chemother 2014, 69:219-227. doi:10.1093/jac/dkt310.

14. Ris-Stalpers $\mathrm{C}$, Bikker $\mathrm{H}$ : Genetics and phenomics of hypothyroidism and goiter due to TPO mutations. Mol Cell Endocrinol 2010, 322:38-43. doi:10.1016/j.mce.2010.02.008.

15. Spitzweg C, Morris JC: Genetics and phenomics of hypothyroidism and goiter due to NIS mutations. Mol Cell Endocrinol 2010, 322:56-63. doi:10.1016/j.mce.2010.02.007.

16. Persani L, Calebiro D, Cordella D, Weber G, Gelmini G, Libri D, de Filippis T, Bonomi M: Genetics and phenomics of hypothyroidism due to TSH resistance. Mol Cell Endocrinol 2010, 322:72-82. doi:10.1016/j.mce.2010.01.008.

17. Cooper DS, Biondi B: Subclinical thyroid disease. Lancet 2012, 379:1142-1154. doi:10.1016/50140-6736(11)60276-6.

18. Au WY, Hawkins BR, Chan EY, Lie AK, Kung AW, Liang R, Cheng N, Kwong YL: Association of the HLA A2-B46-DR9 haplotype with autoimmune thyroid dysfunction after bone marrow transplantation in Chinese patients. Br J Haematol 2001, 115:660-663.

19. Tran HA: Reversible hypothyroidism and Whipple's disease. BMC Endocr Disord 2006, 6:3. doi:10.1186/1472-6823-6-3.

20. Dobbins WOIII: Whipple's Disease. Illinois: Springfield, Thomas Book; 1987.

21. Edouard S, Fenollar F, Raoult D: The rise of Tropheryma whipplei: a 12- year retrospective study of PCR diagnoses in our reference center. J Clin Microbiol 2012, 50:3917-3920. doi:10.1128/JCM.01517-12

22. Fenollar F, Laouira S, Lepidi H, Rolain JM, Raoult D: Value of Tropheryma whipplei quantitative polymerase chain reaction assay for the diagnosis of Whipple disease: usefulness of saliva and stool specimens for first-line screening. Clin Infect Dis 2008, 47:659-667. doi:10.1086/590559.

23. Gonzalez-Galarza FF, Christmas S, Middleton D, Jones AR: Allele frequency net: a database and online repository for immune gene frequencies in worldwide populations. Nucleic Acids Res 2011, 39:D913-D919. doi:10.1093/ nar/gkq1128.

24. Goichot B, Pearce SH: Subclinical thyroid disease: time to enter the age of evidence-based medicine. Thyroid 2012, 22:765-768. doi:10.1089/ thy.2012.2208.com

25. Goichot B, Vinzio S: Subclinical thyroid disorders. Lancet 2012, 380:335-337. doi:10.1016/50140-6736(12)61240-9.

doi:10.1186/1471-2334-14-370

Cite this article as: Lagier et al:: Common subclinical hypothyroidism during Whipple's disease. BMC Infectious Diseases 2014 14:370.

\section{Submit your next manuscript to BioMed Central and take full advantage of:}

- Convenient online submission

- Thorough peer review

- No space constraints or color figure charges

- Immediate publication on acceptance

- Inclusion in PubMed, CAS, Scopus and Google Scholar

- Research which is freely available for redistribution

Submit your manuscript at www.biomedcentral.com/submit 